\title{
MUSCLE DISEASES
}

\section{MRI IN CONGENITAL MYOTONIC DYSTROPHY}

The results of CT and MRI of the brain were analyzed and neurological development was assessed from the neonatal period in 7 children with congenital myotonic dystrophy aged 2-8 years at the Division of Neurology, Chiba Children's Hospital and Department of Pediatrics, Chiba University School of Medicine, Chiba, Japan. Ventricular dilatation seen on the first day of life in 2 of 3 infants had not progressed on follow-up CTs at intervals of 1-6 years. Areas of periventricular hyperintensity on T2 weighted MRIs were seen in all children, and an asphyxial episode at birth was responsible for the MRI abnormalities. Low developmental quotients ranging from 12 to 72 were not correlated with the extent of the periventricular hyperintensity or the ventriculomegaly (Tanabe $\mathrm{Y}$ et al. Neuroradiological findings in children with

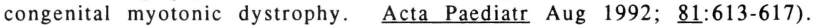
(Correspondence: Dr. Y. Tanabe, Division of Neurology, Chiba Children's Hospital, 579-1 Heta-cho, Chiba, Japan 280-02.)

COMMENT. The mental retardation commonly found in children with congenital myotonic dystrophy was not attributable to brain damage due to perinatal asphyxia. There was no significant correlation between the severity of mental impairment and the degree of structural change in the brain assessed by periventricular hyperintensity on the MRI.

The frequency of cerebral atrophy, large ventricles, and dysmyelination in patients with Fukuyama's congenital muscular dystrophy and occidental congenital muscular dystrophy are compared in a study from Riyadh, Saudi Arabia (Cook J, Gascon G et al. Ann Neurol Sept 1992; 32:439 (abstr)). In 37 children with Fukuyama's congenital muscular dystrophy, $75 \%$ were mentally retarded, $62 \%$ had cerebral atrophy, $81 \%$ large ventricles and $53 \%$ dysmyelination. The mental retardation in this disorder is attributed to a brain dysgenesis.

\section{WERDNIG-HOFFMANN: DOMINANT INHERITANCE}

A family in which both Werdnig-Hoffmann disease and chronic distal spinal muscular atrophy occurred, with apparent autosomal dominant inheritance, is reported from the Department of Neurology, Mayo Clinic Jacksonville, FL and Johns Hopkins University School of Medicine, Baltimore, MD. The female proband developed symptoms of WerdnigHoffmann disease at 2 months of age and died at 10 months. The proband's father and his 2 brothers developed bilateral progressive atrophy and weakness of the hands and legs in their second decade of life. The mother had no symptoms or signs of motor neuron disease, but EMG revealed distal denervation of the limbs. Family studies suggested autosomal dominant inheritance, although the Werdnig-Hoffmann disease may have been influenced by a maternally derived trait. (Boylan KB, Cornblath DR. WerdnigHoffmann Disease and chronic distal spinal muscular atrophy with apparent 